MINI-SYMPOSIUM

\title{
Free radicals and redox signalling in cardiovascular disease
}

\author{
A M Shah, K M Channon
}

Heart 2004;90:486-487. doi: 10.1136/hrt.2003.029389

$\mathrm{T}$ he generation of reactive oxygen species (ROS) is an inevitable consequence of life in an aerobic environment. ROS are characterised by their high chemical reactivity and include both free radicals (that is, species with one or more unpaired electrons, such as superoxide $\left(\mathrm{O}_{2}{ }^{-}\right)$and hydroxyl radicals $\left(\mathrm{OH}^{*}\right)$ ), and non-radical species such as hydrogen peroxide $\left(\mathrm{H}_{2} \mathrm{O}_{2}\right)$. In health, there is a balance between ROS generation and the activity of enzymatic and non-enzymatic antioxidant systems that scavenge or reduce ROS concentrations. ${ }^{1}$ Redox imbalance caused by increased ROS production and/or reduced antioxidant reserve causes oxidative stress-that is, an enhanced susceptibility of biological molecules and membranes to reaction with ROS.

Traditionally, oxidative stress has been considered deleterious due to free radical induced oxidation and damage of macromolecules, membranes and DNA. ROS generation by phagocytic cells such as neutrophils is a pivotal component of their antimicrobial actions and as such deleterious for ingested organisms, but is, however, clearly beneficial for the host. On the other hand, the restoration of oxygen supply during myocardial reperfusion after prolonged ischaemia is accompanied by a burst of free radical production that is damaging for the heart. ${ }^{2}$ Oxidative stress induced damage includes acceleration of cell death through apoptosis and necrosis, mechanisms that may also be of relevance in advanced heart failure.

Recently, however, it has been recognised that oxidative stress may exert more subtle modulatory effects. Firstly, the tightly regulated production of ROS can modulate the activity of diverse intracellular molecules and signalling pathways (a mechanism commonly termed "redox signalling"), with the potential to induce highly specific acute and chronic changes in cell phenotype. ${ }^{3}$ This use of oxygen species to transmit biological information provides at least a teleological explanation for the widespread occurrence of ROS even in health. Secondly, inactivation of the signalling molecule nitric oxide (NO) by ROS is recognised to be a key mechanism underlying reduced NO bioavailability and the development of endothelial dysfunction, which may itself be an important contributor to disease pathophysiology. ${ }^{4}$ In fact, there are interesting parallels between the dichotomous biological activities of $\mathrm{NO}$ and ROS as, on the one hand, signalling molecules and, on the other, critical for immune defence. Excessive unregulated production of either NO or ROS may lead to damaging consequences, while if both are present in excess the generation of peroxynitrite may have even worse effects. ${ }^{4}$

\section{MECHANISMS OF REDOX SIGNALLING}

Redox signalling plays a pivotal role in many disorders-for example, vascular smooth muscle proliferation, atherosclerosis, angiogenesis, cardiac hypertrophy, fibrosis, remodelling. ${ }^{3}$ ROS may induce acute (that is, over seconds to minutes) alterations in cellular functions via specific covalent modifications of target molecules. For example, key proteins involved in myocardial excitation-contraction coupling, such as sarcolemmal ion channels, sarcoplasmic reticulum calcium release channels, and contractile proteins, can all undergo redox sensitive alterations in activity. ${ }^{5}$ ROS also exert important acute effects on cellular energetics. ${ }^{6}$ Chronic changes (that is, over hours and days) in cell phenotype result from modulation of intracellular signalling pathways, such as mitogen activated protein kinases (MAPKs), and redox sensitive transcription factors (for example, NF-kB, HIF-1, AP-1), with consequent alterations in gene and protein expression. ${ }^{3}$ Specificity of signalling derives both from upstream ligand dependent stimulation of ROS production by different enzymatic sources and the targetting of specific downstream pathways by ROS. A wide variety of genes are redox sensitive through the above pathways, depending upon the context-for example, VCAM-1, MCP1, PAI-1, and PDGF during endothelial cell activation.

A fundamental question in the field is to understand the biochemical mechanisms underlying ROS dependent signalling, which may open the way to identifying new therapeutic targets. A common mechanism involves redox dependent covalent modification of specific cysteine residues on target proteins. In the case of tyrosine phosphatase, reversible oxidation of a cysteine residue leads to enzyme inactivation and a secondary increase in activity of tyrosine kinases (for example, specific MAPKs). ${ }^{3}$ A different kind of modification involves the reversible covalent addition of glutathione to cysteine residues (or S-glutathiolation). ${ }^{7}$ Alternatively, oxidation of cellular proteins known as redoxins, which are often associated with signalling molecules, may lead to the activation of such molecules-for example, the MAPKK kinase ASK1. ${ }^{3}$ More recently, novel mechanisms involving the redox dependent release of diffusible factors (termed "secreted oxidative stress induced factors" or SOXF) that induce alterations in gene expression through autocrine and paracrine actions have been described. The accompanying article by Jin and Berk ( $p$ 488) reviews underlying mechanisms of redox signalling with specific focus on the role of SOXF.

\section{SOURCES OF ROS}

The sources of ROS generation in different disease settings are of great interest, especially in relation to redox signalling where the specificity that is essential for biological signalling may derive at least in part from the properties and regulation of the ROS source(s). Potential ROS sources include mitochondria, xanthine oxidase, cytochrome $\mathrm{P} 450$ based enzymes, NADPH oxidases, dysfunctional NO synthases (discussed later), and infiltrating inflammatory cells. The pathological roles of mitochondrial ROS production have been reviewed elsewhere. ${ }^{8}$ Xanthine oxidase derived ROS production is well known to be important in ischaemiareperfusion. More recently, it has been shown that xanthine oxidase can be released into the circulation and bind to the luminal surface of endothelial cells, so that it can exert

Abbreviations: MAPKs, mitogen activated protein kinases; NO, nitric oxide; NOS, nitric oxide synthases; ROS, reactive oxygen species; SOXF, secreted oxidative stress induced factors 
important effects even in tissues where it is not normally expressed. In particular, xanthine oxidase appears to play a role in the genesis of endothelial dysfunction in some settings.

Despite the existence of these multiple sources, a large number of studies in the last decade indicate that a major ROS source involved in redox signalling is a family of complex enzymes termed NADPH oxidases (or Noxs). Although long recognised as being essential for the microbicidal activity of neutrophils, these enzymes are in fact widely expressed in numerous non-phagocytic tissues. They have been shown to play fundamental roles in experimental hypertension, atherosclerosis, endothelial dysfunction, and cardiac hypertrophy, with evidence that different Nox isoforms may have distinct functions. ${ }^{10}$ Recent studies provide confirmatory evidence of similar roles for NADPH oxidases in human disease. ${ }^{91-13}$ The accompanying article by Griendling ( $p$ 491) provides a review of current knowledge regarding the structure and function of Noxs.

\section{ROS PRODUCTION BY DYSFUNCTIONAL NITRIC OXIDE SYNTHASES (NOS)}

NO is generated by NO synthases (NOS) that oxidise the amino acid L-arginine by reduction of molecular oxygen. A central part of our understanding of NO biology and redox signalling revolves around the rapid interactions between NO and ROS such as $\mathrm{O}_{2}{ }^{-}$. The NOS enzymes are complex homodimeric oxidoreductases that shuttle electrons from the reductase domain of one monomer (a cytochrome P450-like region containing the cofactors FAD, FMN, and NADPH) to the oxidase domain in the other subunit that contains the haem active site. Given this enzymatic structure, it is not surprising that recent findings suggest that the NOS enzymes can become "uncoupled", leading to generation of $\mathrm{O}_{2}{ }^{-}$ rather than $\mathrm{NO}$, thus adding additional complexity to the biological relationships between NO, ROS, and redox signalling. ${ }^{4}$

The accompanying article by Verhaar and colleagues ( $p$ 494) discusses the potential importance of $\mathrm{O}_{2}{ }^{-}{ }^{-}$production by NOS. The NOS cofactor tetrahydrobiopterin (BH4) appears to have an important role in regulating NOS function by "coupling" the reduction of molecular oxygen to L-arginine oxidation and in maintaining the stability of NOS dimers. Availability of $\mathrm{BH} 4$, itself a redox active compound that is susceptible to oxidative degradation, may be one mechanism linking oxidative stress to endothelial dysfunction in conditions such as diabetes. ${ }^{14}$ Importantly, initial oxidative loss of $\mathrm{BH} 4$ in response to increased ROS production by NADPH oxidases seems to result in amplification of oxidative stress due to the resulting loss of NO production and increased NOS dependent $\mathrm{O}_{2}{ }^{-}$- generation. ${ }^{15}$ This paradigm illustrates how small changes in ROS production may be amplified and modulated through interactions between different oxidase systems.

\section{THERAPEUTIC RELEVANCE}

What potential therapeutic targets may be identified through understanding redox signalling pathways in the cardiovascular system? Increased oxidative stress plays important roles in numerous pathologies as diverse as heart failure, ischaemia-reperfusion, endothelial dysfunction, hypertension, and atherosclerosis. In several disease settings, treatment with antioxidants or with superoxide dismutase has been shown to be effective in reducing markers of oxidative stress and improving functional parameters such as endothelium dependent relaxation. Despite this clear evidence for the biological importance of ROS, large antioxidant trials have shown no benefit in reducing cardiovascular events or mortality. ${ }^{16}$ However, we should be careful to avoid the spurious conclusion that antioxidant strategies have no place in cardiovascular disease prevention or treatment. Rather, the situation is more complex than initially imagined. Current "antioxidants" are biologically inefficient and unable to target specific redox pathways, although it is clear than some existing drugs already exert some of their effects through redox-dependent signalling pathways (for example, probucol, statins, angiotensin converting enzyme inhibitors and protein kinase $\mathrm{C}$ inhibitors). Future treatments may need to target redox pathways in cell, tissue, and pathway specific manners at an appropriate time in the disease process. The potential utility of existing drugs and the basis for devising novel therapeutic strategies will come from further elucidating detailed mechanisms of action in redox signalling. The accompanying articles in this mini-symposium aim to summarise our current knowledge in working towards a better understanding of some of these mechanisms.

\section{ACKNOWLEDGEMENTS}

The authors' studies are supported by the British Heart Foundation (programme grants RG/03/008 to AMS and RG/02/006 to KMC).

\section{Authors' affiliations}

A M Shah, Department of Cardiology, Guy's King's \& St Thomas's School of Medicine, King's College London, UK

K M Channon, Department of Cardiovascular Medicine, University of Oxford, UK

Correspondence to: Professor A M Shah, Department of Cardiology, GKT School of Medicine, Bessemer Road, London SE5 9PJ, UK; ajay. shah@kcl.ac.uk

\section{REFERENCES}

1 Gutteridge JM, Halliwell B. Free radicals and antioxidants in the year 2000. A historical look to the future. Ann N Y Acad Sci 2000;899:136-47.

2 Ambrosio G, Tritto I. Reperfusion injury: experimental evidence and clinical implications. Am Heart J 1999;138:S69-75.

3 Finkel T. Signal transduction by reactive oxygen species in non-phagocytic cells. J Leukoc Biol 1999:65:337-40.

4 Harrison DG. Cellular and molecular mechanisms of endothelial cell dysfunction. J Clin Invest 1997;100:2153-7.

5 Gao WD, Liu Y, Marban E. Selective effects of oxygen free radicals on excitation-contraction coupling in ventricular muscle. Implications for the mechanism of stunned myocardium. Circ Res 1996;94:2597-604

6 Xie YW, Kaminski PM, Wolin MS. Inhibition of rat cardiac muscle contraction and mitochondrial respiration by endogenous peroxynitrite formation during posthypoxic reoxygenation. Circ Res 1998;82:891-7.

7 Klatt P, Lamas S. Regulation of protein function by S-glutathiolation in response to oxidative and nitrosative stress. Eur J Biochem 2000;267:4928-44.

8 Tsutsui $\mathrm{H}$. Oxidative stress in heart failure: the role of mitochondria. Intern Med 2001;40:1177-82.

9 Spiekermann S, Landmesser U, Dikalov S, et al. Electron spin resonance characterization of vascular xanthine and $\mathrm{NAD}(\mathrm{P}) \mathrm{H}$ oxidase activity in patients with coronary artery disease: relation to endothelium dependent vasodilation. Circulation 2003; 107:1383-9.

10 Byrne JA, Grieve DG, Bendall JK, et al. Contrasting roles of NADPH oxidase isoforms in pressure overload versus angiotensin II-induced cardiac hypertrophy. Circ Res 2003;93:802-5.

11 Guzik TJ, West NEJ, Black E, et al. Vascular superoxide production by $\mathrm{NAD}(\mathrm{P}) \mathrm{H}$ oxidase: association with endothelial dysfunction and clinical risk factors. Circ Res 2000;86:e85-90.

12 Guzik TJ, Mussa S, Gastaldi D, et al. Mechanisms of increased vascular superoxide production in human diabetes mellitus: role of $\mathrm{NAD}(\mathrm{P}) \mathrm{H}$ oxidase and endothelial nitric oxide synthase. Circulation 2002;105:1656-62.

13 Heymes C, Bendall JK, Ratajczak P, et al. Increased myocardial NADPH oxidase activity in human heart failure. J Am Coll Cardiol 2003;41:2164-71.

14 Alp NJ, Mussa S, Khoo J, et al. Tetrahydrobiopterin-dependent preservation of nitric oxide-mediated endothelial function in diabetes by targeted transgenic GTP-cyclohydrolase I over-expression. J Clin Invest 2003; 119:725-35.

15 Landmesser U, Dikalov S, Russ Price S, et al. Oxidation of tetrahydrobiopterin leads to uncoupling of endothelial cell nitric oxide synthase in hypertension. J Clin Invest 2003;111:1201-9.

16 Jialal I, Devaraj S. Antioxidants and atherosclerosis. Don't throw out the baby with the bath water. Circulation 2003;107:926-8. 